\title{
Charge Transfer Evidence in Donor-Acceptor Single-Walled Carbon Nanotubes filled with Sexithiophene Oligomers: Nanotube Diameter Dependence
}

\author{
Chenouf J. $^{1}$ | Boutahir M. ${ }^{1,2 *}$ \\ Rahmani AH. ${ }^{1}$ । \\ Chadli H. $^{1}$ | Patrick Hermet $^{3}$ | Mejía-López J. ${ }^{2}$ । \\ Rahmani A. ${ }^{1}$
}

${ }^{1}$ Laboratoire d'Etude des Matériaux Avancés et Applications (LEM2A), Université Moulay Ismail,FSM-ESTM, BP 11201, Zitoune, 50000 Meknes, Morocco

${ }^{2}$ Centro de Investigación en Nanotecnología y Materiales Avanzados CIEN-UC, Facultad de Física, Pontificia Universidad Católica de Chile. Cedenna, Santiago, Chile

${ }^{3}$ Institut Charles Gerhardt Montpellier, Univ. Montpellier, CNRS, ENSCM, Montpellier, France

\section{Correspondence}

Mourad Boutahir, Centro de Investigación en Nanotecnología y Materiales Avanzados CIEN-UC, Facultad de Física, Pontificia Universidad Católica de Chile. Cedenna, Santiago, Chile

Email: mourad.boutahir@fis.puc.cl

\section{Funding information}

Comisión Nacional de Investigación Científica y Tecnológica (CONICYT)-Fondo Nacional de Desarrollo Científico y Tecnológico (FONDECYT) Posdoctodado 2020 Nº 3200046
Encapsulation of photo-active organic molecules inside single-walled carbon nanotubes (SWNTs), appears to be of great interest in terms of high power conversion effciency, and long-term stability for a commercial application of organic solar cells (OSCs). In this paper, we report a charge transfer (CT) evidence in Donor-Acceptor SWNTs filled with Sexithiophene oligomers (6T) by Raman spectroscopy. To compute the optimal diameter and demonstrate the most stable structure of the hybrid systems with either a single 6T molecule encapsulated into SWNTs (6T@SWNTs), or two 6T chains encapsulated (6T6T@SWNTs), we have performed structural geometry optimization on the hybrid encapsulated systems using a convenient Lennard-Jones (LJ) expression of the van der Waals (vdW) intermolecular potential. Combining the density functional theory (DFT), molecular mechanics, bond polarizability model and the spectral moment method (SMM), we computed the polarized nonresonant Raman spectra of 6T molecule and SWNTs (metallic and semiconducting) before and after encapsulation. The influence of the encapsulation on the Raman-active modes of the $6 \mathrm{~T}$ molecule and those of the nanotube (radial breathing modes and tangential modes) are analyzed. In particular, significant changes observed in the G-band frequency. The possibility (or not) of an eventual CT between the 6T oligomer and the nanotube in both hybrid systems (6T@SWNTs and 6T-6T@SWNTs) is discussed. We show that there is a dependence of the CT with respect to the diameter of SWNTs, the CT vanish with increasing diameter of the nanotubes. Our finding of CT behavior in the the filled SWNTs with respect to SWNT diameter will provide a useful guidance for enhancing the performance of OSCs by SWNTs.

\section{KEY W O R D S}

SWNTs; Sexithiophene; Raman; Charge transfer; Organic solar cells 


\section{I INTRODUCTION}

One of the most effective and practical ways to reduce the current energy crisis and strengthen environmental protection is the exploitation of clean and sustainable solar energy. In this context, photovoltaic (PV) devices, which directly convert solar radiation into electricity, have received more research attention $^{1}$. The conversion and optimisation of sunlight to electricity PCE present the main challenge for PV cell designers. The theoretical upper limit to PCE from a Shockley-Queisser analysis for a single junction solar cell (SC) was reported to be about $30 \%^{2}$.

Currently, silicon-based SCs technology (with a band gap of $1.1 \mathrm{eV}$ ) is by far the dominant PV technology retaining a mature manufacturing and an excellent PV performance with about $25 \%$ efficiency ${ }^{3}$. However, such systems suffer from the high cost of manufacturing and installation, and it is still not cheap enough to globally compete with fossil fuels ${ }^{4}$. Among all the alternative technologies to silicon-based SCs, organic PV have attracted significant attention because of their low costs, simple preparation, large area, mechanical flexibility, and lightweight ability ${ }^{5}$, but the most critical challenges for OSCs are their low efficiency and lifetime ${ }^{6 ; 7}$. Their energy efficiency and durability need to be enhanced for making them practically viable.

Most of the research on OSCs has focused on bulkheterojunction (BHJ) architectures, and one of the most widely examined material systems consisted of conjugated polymer as the electron donor and fullerene derivatives as the electron acceptor ${ }^{8}$. The simple design of these devices typically consist of an active layer comprises an intimate blend of two organic molecular structure, one electron donating strongly light absorbing, and another electron acceptor materials usually a fullerene derivative with electron accepting properties, placed between two electrodes utilized to generate power. In these cells, when light is incident on the device, electron-hole pairs called excitons are formed on the light absorber materials, and then, PV conversion is based on the dissociation of these photo-generated charges into electrons and holes at the donor-acceptor interfaces ${ }^{9}$.

Significant progress has been made for conjugated polymer/fullerene based BHJ OSCs and these typical combinations has afford to PCE close to or exceeding $11 \%{ }^{10}$. Never- theless, the performance of this kind of devices is ultimately limited by the intrinsic defficiencies of fullerene family, including weak absorption in the solar spectrum range, limited optoelectronic tunabilitty, small charge mobility and thermal instability ${ }^{11}$. Thus, alternatives to fullerenes as electron acceptors materials would bring more potential. In this regards, the scientific communities has proposed carbon nanotubes, and in particular, SWNTs as promising alternatives to the fullerenes, because of their several unique structural, excellent photo-chemical stability, mechanical strength, thermal, optical and electronic properties ${ }^{12 ; 13 ; 14 ; 15}$. In comparison with fullerene acceptors, SWNTs possess distinct advantages that are not found in fullerenes, in terms of optical absorption and tunability of bandgaps, it offers distinctive properties such as the very broad spectral coverage with band-gap electronic transitions well into the near-IR spectral region $16 ; 17 ; 18 ; 19$.

Hence, in recent years, SWNTs are considered as attractive candidates and import element in OSCs, and recent trends suggest that a successful utilization of SWNTs, in particular, the direct integration of SWNTs inside the BHJ photo-active layers could potentially overcome many of the handicap and lead to the realization of high efficiency and low-cost OSCs ${ }^{20}$. Indeed, SWNTs offer a wide range of band gaps to match the solar spectrum and enhanced absorption in the IR range (0.48$1.37 \mathrm{eV})^{21 ; 22}$. They also exhibit excellent charge-carrier mobility (up to $10^{5} \mathrm{~cm}^{2} / \mathrm{V} . \mathrm{s}$ ) at room temperature ${ }^{23}$, and enormous current carrying capability (up to $10^{9} \mathrm{~A} \cdot \mathrm{cm}^{-2}$ ) ${ }^{24}$. The use of SWNTs as a light harvester and charge transporter in the active layer of OSCs provides an opportunity to avoiding carrier recombination and permits efficient exciton quenching at the interfaces and charge carrier transport ${ }^{25}$.

In fact, the PCE of OSCs utilizing blends of conjugated polymers and SWNTs as two integrative parts of the photoactive layer is limited to a few percent ${ }^{10}$, and they are still far behind other solar cell technologies, in particular, the fullerene based devices. The limiting factors for SWNTs are several, including the noncomplete exciton-dissociation for the low amounts of SWNTs, and on the other hand, the increased recombination pathways for the high amounts of SWNTs percentage in the polymer matrix ${ }^{14 ; 13}$. Moreover, SWNTs are typically a mixture of metallic and semiconducting as produced nanotubes. This electronic variety may affect the device PCE, and several authors indicate semiconducting nanotubes 
as the only desirable ones for OSCs ${ }^{26 ; 27}$.

Furthermore, although the PCE of OSCs is an important issue for practical applications, the inferior device stability is also necessary in moving these devices toward commercialization, and still remains a great challenge. Many external or intrinsic factors limit OSC stability such as irradiation, heat, mechanical stress, oxygen and humidity ${ }^{7 ; 28 ; 29}$. Concerning the extreme environmental external factors, like oxygen and water, encapsulation strategy for a protection from the ambient could give them a longer stability. As for the internal degradation factors, like irradiation and heating are closely related to the stability of active layer materials. Indeed, intrinsic molecular structure of active layer materials is one of the most important factors that affect the OSCs stability. Furthermore, the degradation of conjugated polymers under illumination is the most challenging handicap to realize long-term OSCs stability. In fact, degradation occurring in OSCs gets accelerated in the presence of UV light and produces the decomposition of organic molecules, and thus results in formation of new molecules with inferior properties, causing degradation in PV performances of the devices. Ensuring, therefore, the stability of the conjugated polymer under continuous light irradiation is critical for achieving practical use of OSCs, and insist on the development of innovative technics allowing to overcome degradation issues.

An elegant solution and powerful technic that can overcomes those several limits consist in encapsulating of photoactive molecules inside SWNTs to form new nanohybrid systems with adjustable optoelectronic properties. These nanohybrid systems are particularly attractive when the preservation of intrinsic SWNTs electronic and charge transport properties is desired, while overcoming the degradation problem of conjugated molecules. Actually, the organic molecules inside SWNTs will be protected from any external reactive species by the surrounding nanotube wall as proposed by Takenobu et $\mathrm{al}^{30}$. In addition, insertion into SWNTs can suppress the light irradiation degradation of the molecules as reported by Yanagi et $\mathrm{al}^{31}$, and thus, the degradation of conjugated molecules might be overcome by encapsulating them inside SWNTs.

Generally, in comparison with conventional fullerene devices, SWNTs filled with organic molecules will possess distinct advantages in terms of high PCEs, and long-term stability for commercial application of OSCs.
In order to complete our study about the encapsulation of a series of oligothiophenes in SWNTs ${ }^{32 ; 33}$, we propose here a comparative investigation on the structural and vibrational properties of nanohybrids formed through SWNTs filled with one of the most important classes of conjugated oligomers that can be applied as photo-active material in OSCs, sexithiophene $(6 \mathrm{~T})^{34 ; 35}$. It is well-known that SWNTs behave not only as electron acceptors but as light-harvesting electron donors as well depending on the electronic nature of the molecule used for their functionalization. Since 6T are electron-donor in nature, SWNTs can act as an acceptor in their donor-acceptor complexes. Indeed, there are several reports on the structure, stability and properties of the filled SWNTs with 6T molecules investigated by using aberration-corrected high-resolution transmission electron microscopy, Raman spectroscopy, and optical spectroscopy ${ }^{36 ; 37 ; 38 ; 39}$. Despite the excellent experimental work in this research field ${ }^{40 ; 41 ; 42}$, leading recently to encapsulate efficiently $6 \mathrm{~T}$ in SWNT at low temperature ${ }^{43}$, computational approaches are indispensable for obtaining atomistic information of 6T inside nanotubes, especially, the close proximity between $6 \mathrm{~T}$ and SWNTs may also result in charge transfer (CT) in the hybrid systems. In the present paper, we theoretically study the CT in the filled SWNTs with 6T molecules using Raman spectroscopy. Firstly, to compute the optimal diameter and demonstrate the most stable structure of the hybrid systems with either one 6T molecule (6T@ @WNTs) and two 6T chains encapsulated (6T-6T@SWNTs)(see figure 1), we have performed structural geometry optimization on the hybrids using a convenient LJ expression of the vdW intermolecular potential. Then, we present calculations of the Raman spectra of 6T molecules and SWNTs before and after encapsulation. The polarized nonresonant Raman spectra are calculated in the framework of the force constants model combined with the spectral moments method (SMM) ${ }^{44}$. Finally, discussion and conclusions in the last section.

\section{2 | MODEL AND METHODOLOGY}

Calculation of Raman spectra necessitates the knowledge of the dynamical matrix (DM) and the polarizability tensor of the system. In our previous works ${ }^{32 ; 33}$, we developed a hybrid approach to compute the nonresonant Raman spectra of 
bi-thiophene (2T) and quater-thiophene (4T) molecules encapsulated into metallic and semiconducting SWNTs, combining the DFT, molecular mechanics, bond polarizability model and SMM. The DM of the hybrid system has a $3 \mathrm{Nx} 3 \mathrm{~N}$ dimension ( $\mathrm{N}$ is the number of atoms in the primitive unit cell). Exploiting the symmetry of the system may be reduced considerably the number of the DM elements to be determined. However, other than the hermitian (lower or upper triangular) character of the DM, in the case of our hybrids, except the trivial identity operation, no symmetry elements exist. Consequently, all the diagonal and off-diagonal elements of the DM have to be calculated.

First principles-based methods are very accurate to compute the DM (frozen phonon or density functional perturbation theory) but their computational cost is very high for large systems, like6T@SWNTs, and especially without any symmetry. Molecular mechanics could be an excellent alternative to build the 6T@SWNTs DM but such a force field still remains unknown. Thus, taking advantage of DFT and molecular mechanics, we adopted a three-step methodology. The idea consists to decompose the 6T@SWNT hybrid system into two independent subsystems (6T and SWNT), which will be coupled with an adequate empirical intermolecular potential. The DM of the 6T@SWNTs hybrids is built block by block from the DM of each subsystem, where the DM of the SWNT and 6T form the bidiagonal block of the DM of the 6T@SWNTs, while the DM associated with the 6T and SWNT interactions forms the off-diagonal block.

In the first step, the DM of the oligomers molecules will be calculated under DFT at the local density approximation (LDA) level of theory, as implemented inside the Spanish Initiative for Electronic Simulations with Thousands of Atoms (SIESTA) package ${ }^{45}$. In the second step, the DM of SWNT will then be computed from the force constant model introduced by Saito et $\mathrm{al}^{46}$. Lastly, the vdW interactions (longranged dispersive forces) are the binding mechanism in the hybrid systems under investigation. LJ potential is adopted for an adequate description of these interactions between 6TSWNTs:

$$
U_{L J}(r)=4 \varepsilon\left[\left(\frac{\sigma}{r}\right)^{12}-\left(\frac{\sigma}{r}\right)^{6}\right]
$$

where : $\epsilon_{c}=2.964 \mathrm{meV}$ and $\sigma_{c}=0.3407 \mathrm{~nm}, \epsilon_{s}=$ $10.7 \mathrm{meV}$ and $\sigma_{s}=0.3560 \mathrm{~nm}$, and $\epsilon_{h}=0.67 \mathrm{meV}$ and $\sigma_{h}=0.1069 \mathrm{~nm}$ are the LJ parameters of carbon, sulfur, and hydrogen atoms respectively. We calculated the LJ parameters of carbon-sulfur and carbon-hydrogen using the combining rules of Lorentz-Berthelot: $\varepsilon_{A B}=\sqrt{\varepsilon_{A} \varepsilon_{B}}$ and $\sigma_{A B}=\left(\sigma_{A}+\right.$ $\left.\sigma_{B}\right) / 2$.

The DFT calculations on 6T molecules have been carried out using LDA exchange-correlation functional. The valence electrons were described by a double- singly polarized basis set. The localization of the basis is controlled by an energy shift of $50 \mathrm{meV}$ and the real space integration was performed on a regular grid corresponding to a plane wave cuto around $360 \mathrm{Ry}$. We considered a vacuum size of $12 \AA$ to avoid interactions between adjacent systems.

The Raman susceptibility tensor is computed using the bond polarizability model of SWNTs ${ }^{47}$ combined with one of the free $6 \mathrm{~T}$ molecules ${ }^{48}$. This approach has been successfully used to predict the nonresonant Raman spectra of SWNTs and double-walled carbon nanotubes (DWNTs) as a function of their lengths $49 ; 50 ; 51 ; 52$. For more details on the Raman equations and the computational details to derive the Raman susceptibility from the bond polarizability model, see ref ${ }^{48}$. Finally, Computation of Raman spectra of the investigated hybrids are based on the SMM. Indeed, the usual method to calculate the Raman response requires a direct diagonalization of the DM of the system. However, when the system under investigation contains a large number of atoms (like 6T@SWNT), the DM is very large and its diagonalization demands a very long computing time or break down. In such cases, the SMM appears as a unique and very powerful method as it allows us to compute directly the Raman spectra without any diagonalization of the DM. For more details on this hermetic method, see Ref ${ }^{44 ; 49}$.

\section{3 | RESULTS AND DISCUSSION}

\section{1 | Stability of 6T confined into SWNTs}

Aiming to gain insight into the formation of the filled nanotubes in terms of bonding, geometry, and stability, we focus here on the structural arrangements of such nanohybrids which are prerequisites for comprehension of their properties. In our 
calculations, we respectively considered two con figurations: the 6T@SWNTs where only one 6T molecule is placed inside the nanotube, and the 6T-6T@SWNTs where two molecules are placed inside the nanotube cavity. We determined the range of nanotube diameters in which the encapsulated systems are stable for the two encapsulated systems (6T@SWNTs and6T-6T@SWNTs). Such a study could lead the experimentalists to provide effective estimates about reasonable and suitable SWNT sizes and the steric hindrance of the 6T in the inner space of the nanotube. In our calculations, we adopt that the sizes of the oligothiophenes are sorely reduced under nanoconfinement by aligning all the $6 \mathrm{~T}$ rings to form a planar structure. For a single 6T molecule placed inside SWNT, the optimized arrangements correspond to an isolated chain inserted inside SWNTs cavity and centered along the nanotube axis. On the other hand, concerning the optimized configuration for two $6 \mathrm{~T}$ molecules, we have first adopted an initial position of $6 \mathrm{~T}$ molecules according to the minimum energy calculations using LJ potential. The interchain distance found for two free-standing 6T molecules in a parallel configuration is $\mathrm{d}=0.508 \mathrm{~nm}$ (see figure 1 ).

(a) Sexithiophene (6T)
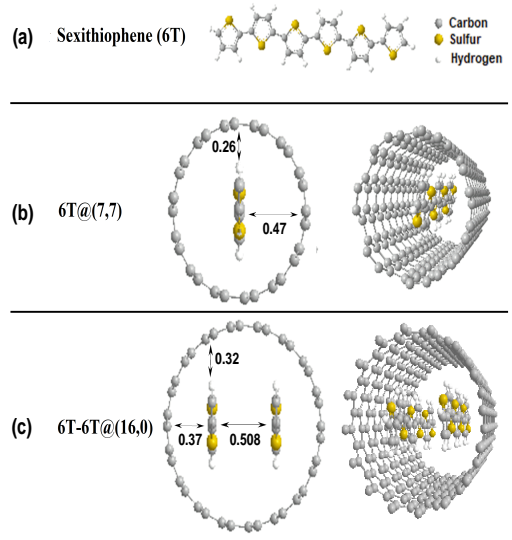

F I G URE 1 Schematic of the encapsulation of 6 T molecule in SWNTs and characteristic distances after energy minimization. (a) 6T, (b)6T@ (7,7) and (c)6T-6T@(16.0). The highlighted distances are given in $\mathrm{nm}$. Atom colors: grey-carbon, yellow—sulfur and white-hydrogen.

In practice, the structure relaxation is performed by minimizing the distance between atoms associated with $\mathrm{LJ}$ poten- tial. Figure 2 shows the LJ potential as a function of SWNT diameter varying from 0.8 to $2.5 \mathrm{~nm}$ and from 1.1 to $2.9 \mathrm{~nm}$ for6T@SWNTs and6T-6T@SWNTs hybrid systems respectively. First, for 6T@SWNT hybrid system, we observe a minimum at $0.93 \mathrm{~nm}$ corresponding to the equilibrium distance between the SWNT and 6T molecule.

Thus, the insertion of a single 6T molecule, located aligned at the SWNT center, is particularly suitable for SWNTs with a diameter around $0.93 \mathrm{~nm}$. Good agreement acquired between this diameter value obtained from the minimization of the LJ energy and the experimental observations of Alvarez et al ${ }^{53}$ for a single oligothiophene molecule. For 6T-6T@SWNTs hybrid systems, figure 2 gives clear evidence that encapsulating two 6T molecules is favorable for tubes with diameter close to $1.24 \mathrm{~nm}$. This optimal diameter agrees well with Loi et $\mathrm{al}^{37}$ experimental observations for two parallel oligothiophene molecules placed inside SWNTs, and the fact that the RBM position in Raman spectra of the SWNTs is at $196 \mathrm{~cm}^{-1}$ (correspond to mean diameters of $1.21 \mathrm{~nm}$ according to the relationship proposed in ref $^{54}$ ).

Beside that, total energy calculations suggest that the vdW interactions between the $6 \mathrm{~T}$ and the tube is stronger than the intermolecular interaction ${ }^{37}$. Coherently, such behavior is reported by yumura et al. ${ }^{55}$ in their investigation on the optimized geometries for terthiophene inside SWNTs armchair, which demonstrate a good correlation to our findings in term of SWNT size and structural arrangement of thiophene oligomers inside SWNT, where the optimal distance of the 6T chain to the tube wall is distinctly smaller than the interchain distance (see figure1). Therefore, the vdW bond between the $6 \mathrm{~T}$ and the tube turns out to be stronger than the intermolecular interaction, and the internal 6T prefer to be located near the walls of the nanotubes rather than close to the axis.

\section{2 | Raman spectra of isolated 6T}

The calculated Raman spectrum of 6T molecule in the 0$1680 \mathrm{~cm}^{-1}$ range is ilustrated in figure 3 . The spectrum shows various lines. The analyzis of the corresponding eigenvectors allowed to their assignements, which are in good agreement with the experimental ones ${ }^{56 ; 57 ; 58}$, except for the lowest frequencies between 0 and $90 \mathrm{~cm}^{-1}$ that are not experimentally available. 


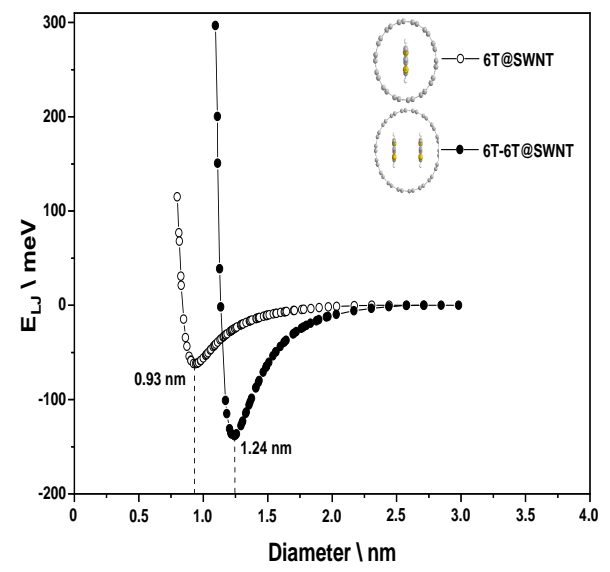

F I G URE 2 Lennard Jones potential as a function of the tube diameter for hybrid systems with one (6T) and two (6T-6T) chains encapsulated in SWNTs.

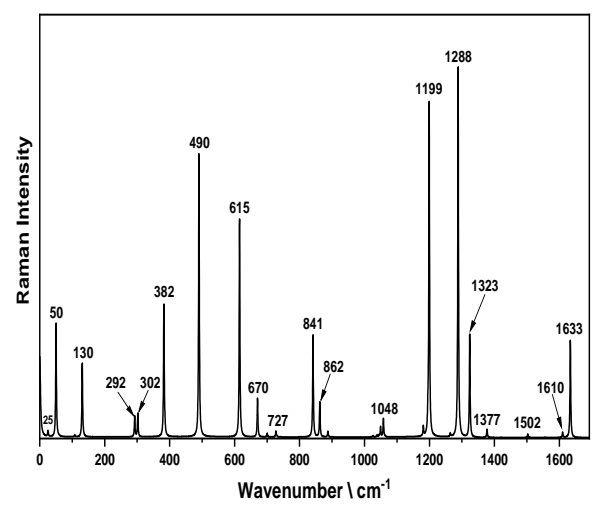

FIGURE 3 Raman spectrum of isolated 6T.

Let us first consider the lower-frequency range $0-400 \mathrm{~cm}^{-1}$. This frequency range is dominated by three features centered at 50,130 and $382 \mathrm{~cm}^{-1}$, two weak lines centered at 8 and $25 \mathrm{~cm}^{-1}$, and one doublet around $295 \mathrm{~cm}^{-1}$. For Raman frequencies below $90 \mathrm{~cm}^{-1}$, no experimental data has found in the literature. Though, three Raman modes are predicted from DFT simulations (two modes centered at 8 and $25 \mathrm{~cm}^{-1}$ and one mode centered at $50 \mathrm{~cm}^{-1}$ ). The couple of lines at 8 and $25 \mathrm{~cm}^{-1}$ are libration modes, whereas the feature located at
$50 \mathrm{~cm}^{-1}$ is associated to in-plan bending (C-S-C) and in-plan bending $(\mathrm{C}-\mathrm{H})$ modes. For the other features at 130, 295 and $382 \mathrm{~cm}^{-1}$ are assigned respectively to the in-plane bending $(\mathrm{S}-\mathrm{C}=\mathrm{C})$ modes, the scissor ( $\mathrm{S}-\mathrm{C}-\mathrm{C}-\mathrm{S})$, and in-plane bending $(\mathrm{S}-\mathrm{C}=\mathrm{C})$ with the contribution of in-plan bending $(\mathrm{C}-\mathrm{H})$ modes.

Next, the intermediate-frequency range $\left(400-1100 \mathrm{~cm}^{-1}\right)$ is dominated by two lines centered at 490, 615 and one doublet around $850 \mathrm{~cm}^{-1}$, three weak features centered at 670, 727, and one doublet around $1048 \mathrm{~cm}^{-1}$. These lines are assigned respectively to the in-plane torsional $(\mathrm{S}-\mathrm{C})$ modes, the in-plane bending $(\mathrm{C}-\mathrm{S}-\mathrm{C})$, the stretchings $(\mathrm{C}-\mathrm{S})$ with the contribution of the in-plane bending $(\mathrm{C}-\mathrm{H})$, the in-plane bending $(\mathrm{C}-\mathrm{S}$ C) combined to the out-of-plane bending $(\mathrm{C}-\mathrm{H})$ modes, a combination of the streachings $(\mathrm{C}-\mathrm{S})$ and the in-plane bending $(\mathrm{C}=\mathrm{C}-\mathrm{C})$, and the stretching $(\mathrm{C}-\mathrm{C})$ modes along with the inplane bendings (C-C-H ).

Now, in the heigher-frequency range $\left(1100-1680 \mathrm{~cm}^{-1}\right)$, four strong lines were observed, centered at 1199, 1288, 1323 and $1633 \mathrm{~cm}^{-1}$, and three weak features located at 1377,1502 , and $1610 \mathrm{~cm}^{-1}$. The modes at $1199 \mathrm{~cm}^{-1}$ along with those at 1288 and $1323 \mathrm{~cm}^{-1}$ are assigned to a progression of the in-plane bendings $(\mathrm{C}-\mathrm{C}=\mathrm{C})$ and the in-plane bendings $(\mathrm{C}$ $\mathrm{H}$ ), whereas a combination of the stretchings (C-C) and the in-plane bendings $(\mathrm{C}=\mathrm{C}-\mathrm{S})$ give rise to the feature located at $1377 \mathrm{~cm}^{-1}$ Finally, the stretching $(\mathrm{C}=\mathrm{C})$ along with the inplane beniding $(\mathrm{C}-\mathrm{S}-\mathrm{C})$ vibrations contribute to the lines centered at frequencies higher than $1400 \mathrm{~cm}^{-1}$.

Finally, the stretching $(\mathrm{C}=\mathrm{C})$ along with the in plane bending (C-S-C) vibrations contribute to the lines centered at frequencies higher than $1400 \mathrm{~cm}^{-1}$. It is worth mentioning that the line around $1500 \mathrm{~cm}^{-1}$ which is a common feature of the Raman spectra of aromatic and heteroaromatic systems, where both the $\mathrm{C}=\mathrm{C}$ and $\mathrm{C}-\mathrm{C}$ stretching vibrations are active, is always very strong and dominates the whole Raman spectrum. While this line is calculated at $1502 \mathrm{~cm}^{-1}$ with small intensity, which it can be explained necessarily as a coupling between the $\mathrm{C}-\mathrm{C}$ bonds motions and the in-plane bending $(\mathrm{C}-\mathrm{H})$ whither the hydrogen atoms recoil with a large vibrational displacement which opposes that of the carbon atoms, leading thus to a losing of its intensity. 


\section{3 | Raman spectra of 6T molecules confined into SWNTs}

A Raman spectrum of pristine SWNTs comprises two main characteristic bands: the lower frequency radial breathing modes (RBM) (100- $\left.400 \mathrm{~cm}^{-1}\right)$, which corresponds to coherent vibrations of the all carbon atoms in the radial direction, and the heigher frequency tangential modes (TM) or G-band (above $1500 \mathrm{~cm}^{-1}$ ), which belongs to longitudinal and transversal vibrations of carbon atoms ${ }^{59}$. The investigation of Raman spectra changes induced by the filling of SWNTs with $6 \mathrm{~T}$ molecules is of special interest in the nanotube community. Indeed, changes in the Raman spectra allow monitoring structural modifications of the nanotube sidewalls due to CT effects ${ }^{60}$. This CT effect resulting from the $6 \mathrm{~T}$ molecules encapsulated inside SWNT can be probed through the downshifts and (or) up-shifts observed in the Raman modes, and a survey of this phenomenon (The possibility (or not) of a CT) constitutes the main goal of the present investigation.
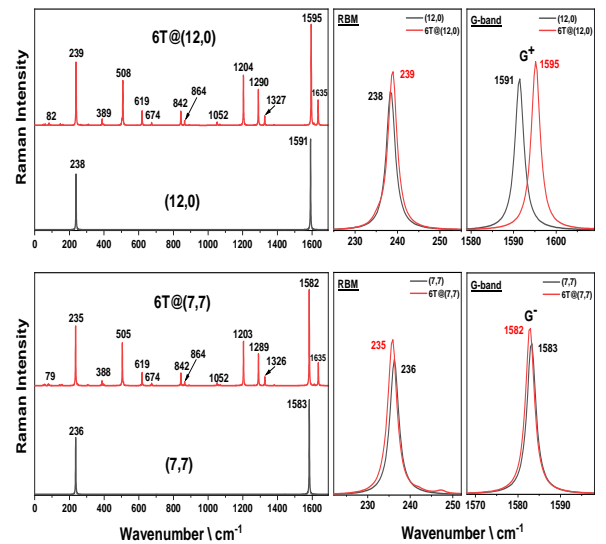

F I G U R E 4 Raman spectra of the empty (black curves) and encapsulated metallic SWNTs (red curves).(top) $(12,0)$ and6T@(12,0). (bottom) $(7,7)$ and 6T@ $(7,7)$.

For this purpose, first, we considered four 6T@SWNT hybrids with different nanotube chirality (n, m),possessing a similar diameter to one another, around the optimal value $(0.93 \mathrm{~nm})$ found in section 3.1 to encapsulate a single $6 \mathrm{~T}$. Amongst, two are metallic $[(12,0)$ and $(7,7)]$ with diameters near to $0.93 \mathrm{~nm}$, and two are semiconducting $[(8,6)$ and $(10,3)]$ (0.95nm and $0.92 \mathrm{~nm}$ in diameter respectively). The ZZpolarized Raman spectra of empty SWNTs and 6T@SWNTs hybrids after minimizations are displayed in figures 4 (metallic tubes) and 5 (semiconducting tubes). Next, we considered four6T-6T@SWNTs hybrids with different tube chirality (n, $\mathrm{m}$ ) and having a similar diameter to one another, around the optimal value obtained $(1.24 \mathrm{~nm})$ to encapsulate two molecular chains (6T-6T). Amongst, two are metallic [(12,6) and $(9,9)](1.24 \mathrm{~nm}$ and $1.22 \mathrm{~nm}$ in diameter respectively), and two are semiconducting $[(16,0)$ and $(13,5)]$ with diameters close to $1.25 \mathrm{~nm}$. The ZZ-polarized Raman spectra of empty SWNTs and6T-6T@SWNTs hybrid systems after minimizations are displayed in figures 6 (metallic tubes) and 7 (semiconducting tubes). For each SWNT,6T@SWNT, and 6T-6T@SWNT the intensities of the different spectra are normalized and can be thus compared.
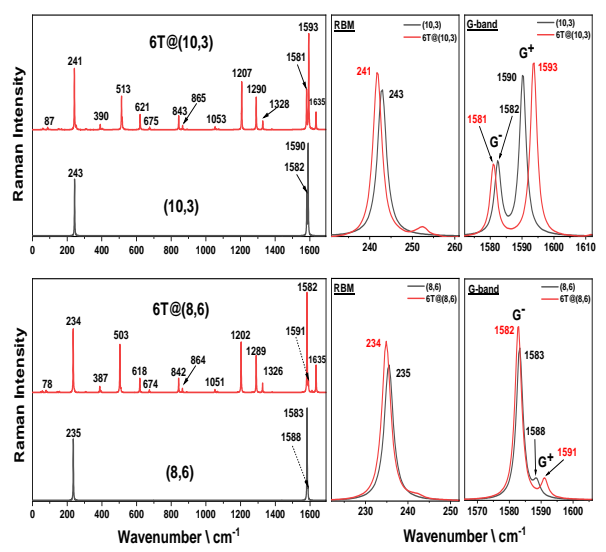

F I G URE 5 Raman spectra of the empty (black curves) and encapsulated semiconducting SWNTs (red curves).(top) $(10,3)$ and 6T@ $(10,3)$. (bottom) $(8,6)$ and 6T@ $(8,6)$.

\subsection{1 | Raman spectra of 6T @SWNTs}

Figures 4 ( metallic SWNTs) and 5 (semiconducting SWNTs) demonstrate the Raman spectra of the 6T@ SWNTs hybrid systems (red curves) in comparison with the spectra of the empty SWNTs (black curves). It is visible that all spectra of the 6T@SWNTs hybrids apear as the superposition of the 6T and SWNTs spectra whatever the electronic charateristic of the 
nanotube (semiconducting or metallic). However, a more careful comparison of Raman spectra shows that the encapsulation leads to the significant modifications of Raman modes. The fitting with individual components of the RBM and G-bands modes permits investigating in detail the observed modifications which are characteristic of the interactions between the 6T and SWNTs in 6T@SWNTs encapsulated systems.
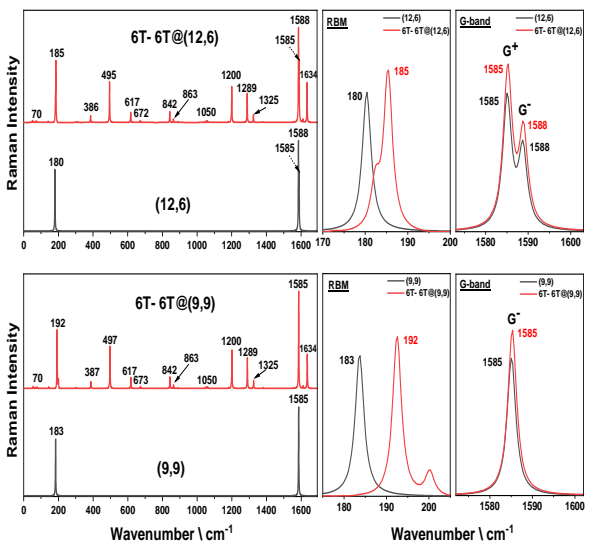

F I G U R E 6 Raman spectra of the empty (black curves) and encapsulated metallic SWNTs (red curves).(top) $(12,6)$ and 6T-6T@(12,6). (bottom) $(9,9)$ and 6T-6T@ $(9,9)$.

Concerning the main modes of 6T oligomer, Raman spectra of 6T@SWNTs hybrid systems with metallic (figure 4) or semiconducting (figure 5) nanotubes show an up-shift due to $\mathrm{vdW}$ interactions, as well as the disappearance of the other lines. Indeed, the lines located in the lower frequency range are very sensitive to the confinement of $6 \mathrm{~T}$ into SWNT.

It is visible that the libration modes $\left(8\right.$ and $\left.25 \mathrm{~cm}^{-1}\right)$ of $6 \mathrm{~T}$ molecule appear in figure 3 vanish in the Raman spectra of 6T@SWNTs hybrids shown in figures 4 and 5. This absence of libration clarify a stability of $6 \mathrm{~T}$ in the SWNT cavity. The free 6T with its fixed orientation repeatedly rotates slightly back and forth, then this reciprocating motion is deactivated after encapsulation, and consequently cause the absence of libration modes. Besides, for the different SWNTs considered, an up-shift associated to vdW interaction of all Raman features between 1 and $23 \mathrm{~cm}^{-1}$ is spotted on the 6T@SWNTs spectra after encapsulation with respect to corresponding ones in the free 6T molecule, which constitutes another hint of a sig- nificant interaction between the subsystems (6T and SWNT) in the hybrids.

In the low-frequency range $\left(100-400 \mathrm{~cm}^{-1}\right)$, encapsulation of 6T into SWNT is expected to induce a certain shift in the RBM band positions of 6T@SWNT, since encapsulated oligomers should affect the motion of carbon atoms in the nanotube along its radius, mainly the RBM shifts induced under the nanoconfinement effect can originate from dispersive interactions (vdW) and CT. Indeed, for the fullerenes-filled SWNTs $61 ; 62 ; 63 ; 64 ; 65 ; 66$. the dispersive interactions (vdW) induce a small down-shift in RBM band of SWNTs of 2 or $3 \mathrm{~cm}^{-1}$. Bandow et al ${ }^{67}$ experimentally showed that fullerenes encapsulated into SWNTs caused a down-shift in the RBM of nanotubes by encapsulation, the authors explain this downshift in RBM band by softening of the C-C bonds of the SWNT sidewall, corresponding to exothermic fullerene encapsulation. Nevertheless, Okada and co-workers ${ }^{68}$ predicted a frequency shift of RBM phonon for the $\mathrm{C} 60$ molecules inserted into metallic armchair SWNTs depending on the tube diameter where the encapsulation of C60 molecules gave a higher frequency shift for $(10,10)$ SWNT but a lower shift for $(11,11)$ and $(12,12)$ SWNTs with greater diameters. This diameter dependence of the frequency shift is ascribed to the charge redistribution induced by weak hybridization between $\pi$-electron states and nearly free electron states. In addition, the small shift of the RBM band to a higher frequency indicates that, energetically, the SWNT diameter is favorable for the molecules encapsulated ${ }^{68}$. On the other hand, CT to and from SWNT can modify the $\mathrm{C}-\mathrm{C}$ bonds in the nanotube and therefore the SWNT electronic properties. Doping SWNTs with strong electron-donor or acceptor constitutes a direct way to observe the CT effect. The insertion of alkali metals (electron-donors) species into SWNTs, rubidium ${ }^{69}$ for example, no shift in the RBM is observed, and a small down-shift for lithium ${ }^{70}$. By contrast, Chen et al. ${ }^{71}$ reported that doping of SWNTs with caesium gives rise to an upshift in RBM by about $2 \mathrm{~cm}^{-1}$. This behavior is observed also when electron acceptor molecules are encapsulated into bundles of SWNTs $^{72}$. However, the CT (positive or negative) onto individual metallic nanotubes ${ }^{73}$ has been reported to induce a small RBM upshift of about $2 \mathrm{~cm}^{-1}$. Such behavior is observed when oligothiophene derivatives $37 ; 74 ; 75$ are inserted into bundles of carbon nanotubes. It was surprising that the 
same direction of RBM shift is seen for SWNTs doped with electron-donor and acceptor, making RBM shifts as a measure of charge transfer ambiguous without prior knowledge of the ionization potential of the molecule or electron affinity. Generally, the RBM shifts are at most $-3 \mathrm{~cm}^{-1}$ for dispersive interactions ${ }^{68 ; 72 ; 76}$ and $2 \mathrm{~cm}^{-1}$ or $\mathrm{CT}^{71 ; 73}$. Thus, for semiconducting6T@SWNTs, the peak positions in empty SWNTs are downshifted by $2 \mathrm{~cm}^{-1}$ and $1 \mathrm{~cm}^{-1}$ for 6T@(10,3) and $6 \mathrm{~T} @(8,6)$ respectively (figure 5$).$ As well, the peak positions in the empty armchair $(7,7)$ metallic tube is down-shifted by about $1 \mathrm{~cm}^{-1}$ in6T@(7,7) spectrum, whereas for6T@(12,0) hybrid, the RBM clearly exhibits a frequency up-shift after encapsulation by $1 \mathrm{~cm}^{-1}$ (figure 4). However, the physical interactions between 6T oligomers and the SWNTs are presumably dispersive and combined to a CT as suggested by Alvarez et al $^{53}$ for the oligothiophene derivatives filled carbon nanotubes, where both effects may compensate each other, and the shift of the RBM is expected to be smaller or close to zero.

Concerning the high-frequencies region where the TM are observed, the G-band feature for pristine SWNTs is fitted with two main components. The lower frequency peak $\left(G^{-}\right)$ is assigned to the transverse optical (TO) phonon modes, and the other peaked at a higher frequency $\left(G^{+}\right)$belongs to the longitudinal optical (LO) phonon modes, independently of whether SWNT is metallic or semiconducting. The chirality dependence of TM where the $G^{-}$peak (resp. $G^{+}$) is dominant at the armchair chiral angle $\left(\theta=30^{\circ}\right)$ (resp. zigzag chiral angle $\left.\left(\theta=0^{\circ}\right)\right)$, and the appearance of two peaks $\left(G^{-}\right.$and $\left.G^{+}\right)$when increasing from $0^{\circ}$ to $30^{\circ}$ was discussed in detail in $\operatorname{Ref}{ }^{49}$. Another point to mention is the electron-phonon coupling which gives rise to the Breit Wigner-Fano lineshape observed in metallic SWNT ${ }^{77}$, does not considered in the present approach. Consequently, the spectra exhibit only Lorentzian lineshapes. The splitting of the $G^{-}$and $G^{+}$Raman peaks (two narrow Lorentzian peaks), and their assignment to the TO and LO phonons, respectively, was discussed in our previous work ${ }^{33}$.

For the G-band of metallic SWNTs (right-hand part of figure 4), the calculated Raman spectra are featured by an only peak affected to $A_{1 g}(\mathrm{ZZ}) \mathrm{TM}$, peaked at $1591 \mathrm{~cm}^{-1}$ in the $(12,0)$ zigzag SWNT, and at $1582 \mathrm{~cm}^{-1}$ in the $(7,7) \mathrm{arm}-$ chair SWNT. It is visible that the G-band spectra of the filled
SWNTs as compared to the pristine SWNTs demonstrate a down-shift of the $G^{-}$peak (TO mode) by $1 \mathrm{~cm}^{-1}$ in the $6 \mathrm{~T} @(7,7)$, as well as a significant up-shift of the $G^{+}$peak (LO mode) by $4 \mathrm{~cm}^{-1}$ in the 6T@(12,0) spectrum. For the G-band of semiconducting SWNTs (right-hand part of figure $5)$, the two inspected tubes $(10,3)$ and $(8,6)$ are chiral with $\theta$ $\simeq 13^{\circ}$, and $25^{\circ}$, respectively, and both the $G^{-}$and $G^{+}$peaks appear in the Raman spectra of empty SWNTs. Under confinement effect, the spectra of the filled SWNTs show a down-shift of the $G^{-}$peaks (TO modes) by $1 \mathrm{~cm}^{-1}$ At the opposite, the $G^{+}$peaks (LO modes) demonstrates a considerable up-shift by $3 \mathrm{~cm}^{-1}$ for the 6T@ $(10,3)$ and 6T@ $(8,6)$ hybrids.

On the basis of these observations, and as previously was reported for the 2T@SWNTs ${ }^{32}$ and 4T@SWNTs ${ }^{33}$ hybrid systems, the G-band behavior under the confinement effect for both metallic and semiconducting6T@SWNTs hybrids shows also similar trends. The $G^{-}$peaks (TO) of SWNTs show a slight down-shift of about $1 \mathrm{~cm}^{-1}$ whereas the $G^{+}$peaks (LO) exhibit a larger up-shift by about 3 and $4 \mathrm{~cm}^{-1}$.

Indeed, the $G^{+}$feature associated with axial carbon atom vibrations (LO) of the SWNT (see figure 8) is sensitive to CT from dopant additions to SWNTs ${ }^{78 ; 79}$. It was discussed that the up-shift of the G-band lines corresponded to the CT from the SWNTs to the encapsulated molecules (p-doping) ${ }^{30 ; 80 ; 81}$ and the down-shift of the G-band lines testified to the CT from the incorporated molecules to the SWNT walls (ndoping $)^{30 ; 80 ; 82}$. Further, it was reported that p-doping leads to the contraction of bonds and phonon stiffening, consequently, this change of the $\mathrm{C}-\mathrm{C}$ bond length under $\mathrm{CT}$ leads to an upshift of the $G^{+}$band ${ }^{83}$. However, Kalbac et al ${ }^{84}$ showed that applying the positive potentials to the SWNTs (analogous to n-doping) may also induce the hardening of the G-band. That is why conclusions about the direction of CT in our hybrid systems cannot be drawn exclusively from Raman spectra. Fortunately at all events, we can conclude that these up-shifts of the $G^{+}$lines proved a CT takes place between the SWNTs and $6 \mathrm{~T}$ molecules.

On the other hand, for the insignificant shift of the $G^{-}$ peaks associated with circumferential carbon atoms vibrations (TO) of the SWNT (see figure 8) toward lower frequencies, there is possibly a concurrence between dispersive interactions of 6T oligomers with SWNTs which give rise to phonon softening and the shift of the $G^{-}$peaks toward lower frequen- 
cies, and the effect of a CT which results in the up-shift of the $G^{-}$peaks. Consequently, due to the compensation of the both effects, it is expected that the shifts of the $G^{-}$peaks should be smaller or even to have the opposite sign than for p-doping.

\subsection{2 | Raman spectra of 6T-6T@SWNTs}

Our discussion up to now referred to a single 6T molecule encapsulated into a SWNTs. In this section, we want to explore the Raman responses of carbon nanotubes filled with more than one molecule. The configuration inspected in this case consists in two molecular chains inserted in the same SWNT. Thus, we considered four 6T-6T@SWNT hybrids with different tube chirality $(n, m)$ and whose the diameters are around $1.24 \mathrm{~nm}$ (the optimal value found in section 3.1 to encapsulate two 6T). The Raman spectra of the 6T-6T @SWNTs hybrid systems (red curves) in comparison with the spectra of the empty SWNTs (black curves) are displayed on figures 6 and 7 respectively. Likewise, all spectra of the 6T-6T@SWNTs hybrids show a superposition of the 6T and SWNTs spectra whatever the electronic charateristic of the nanotube (semiconducting or metallic). However, the detailed analysis of RBM and G-bands modes, as well as the Raman modes of the oligomers allows obtaining further report about the CT in the encapsulated SWNTs.
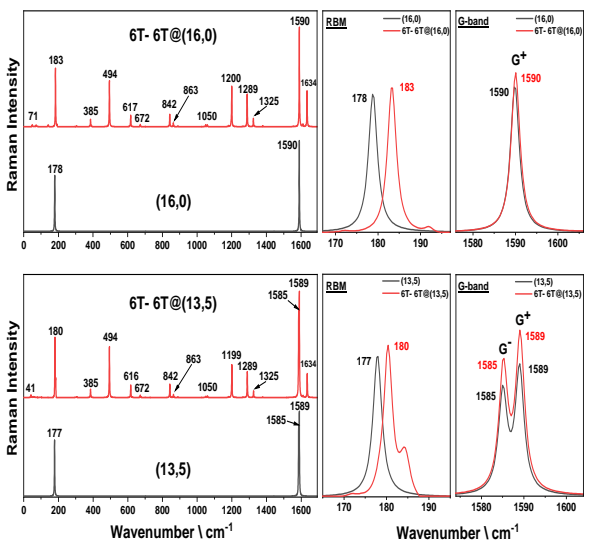

F I G U R E 7 Raman spectra of the empty (black curves) and encapsulated semiconducting SWNTs (red curves).(top) $(16,0)$ and 6T-6T@ $(16,0)$. (bottom) $(13,5)$ and 6T-6T@(13,5).
First, concerning the modes of encapsulated oligothiophene molecules, Raman spectra of 6T-6T@SWNTs hybrids with metallic (figure 6) or semiconducting (figure 7) nanotubes show an up-shift due to vdW interactions, as well as the vanishing of other modes. The libration modes located in the lower frequency region ( 8 and $25 \mathrm{~cm}^{-1}$ ) disappear in the Raman spectra of 6T-6T@ @WNTs hybrids (figures 6 and 7). The vanishing of the librations explicates the stability of confined 6T-6T inside the SWNTs cavity as discussed for nanotubes filled with only a single molecule. Further, an up-shift of the $6 \mathrm{~T}$ mode frequencies (from 1 to $7 \mathrm{~cm}^{-1}$ ) in 6T-6T@SWNTs spectra as compared to the corresponding positions in the isolated 6T molecule (figure 3), indicates a considerable interaction between 6T-6T and the nanotubes under confinement effect.

Next, in the RBM frequency region $\left(100-400 \mathrm{~cm}^{-1}\right)$, all filled nanotubes exhibit an up-shift in the RBM frequency after encapsulation as shown in figures 6 and 7. Indeed, for 6T-6T@SWNTs with metallic nanotubes, the RBM observed in empty tubes is up-shifted by about $5 \mathrm{~cm}^{-1}$ or 6T-6T@ $(12,6)$ and by about $9 \mathrm{~cm}^{-1}$ for 6T-6T@(9,9) (figure 6). Likewise, in the case of semiconducting nanotubes, the RBM observed in empty SWNTs is up-shifted by $5 \mathrm{~cm}^{-1}$ for 6T6T-@ $(16,0)$, and by $3 \mathrm{~cm}^{-1}$ for 6T-6T@ $(13,5)$ (figure 7). Actually, experimental observation of Water filling of thin SWNT down to chiral index $(5,3)$ and theoretical studies on encapsulation of sexithiophene in SWNTs suggesting that the RBM behaviors can be associated to the amount of confined molecules into SWNTs and their supramolecular organization ${ }^{85}$. However, the encapsulation of Zn-diphenylporphyrin (electron donor) inside SWNTs leads to an RBM up-shift of 3 to $5 \mathrm{~cm}^{-1}$ and intensity reduction, which was interpreted by Kataura et al ${ }^{86}$ as a sidewall deformation due to the large size of the molecule (about $1.0 \mathrm{~nm}$ ).

A comparison with the detailed study reported by Almadori et al $^{74}$ about the RBM shift behaviour with a nanotube diameter dependence for the encapsulation of different kinds of oligothiophenes (quaterthiophene, dimethylquaterthiophene, and sexithiophene), revealed an agreeable similarity on the behavior of the RBM shifts observed in both our hybrid systems (6T@SWNTs and 6T-6T@SWNTs). Indeed, for small diameter SWNTs (between 0.68 and $1.1 \mathrm{~nm}$ ) where only one single molecule can fit in the tube, the shifts 
of the RBM mode are almost constant (around a value of 2 $\mathrm{cm}^{-1}$ ). Then, for nanotube diameters between 1.10 and 1.45 $\mathrm{nm}$, which can accommodate two molecules, a linear behavior of the RBM up-shits is observed (from 4 to $8 \mathrm{~cm}^{-1}$ ). These RBM behavior suggests that the physical interactions of thiophene molecules with the largest diameter nanotubes (larger than $1.1 \mathrm{~nm}$ ), cannot be simply understood as a superposition of dispersive interactions and CT, but it can be understood as a mechanical strain on SWNTs induced by the confined 6T-6T molecules (as evidenced in ref. ${ }^{74}$ ).

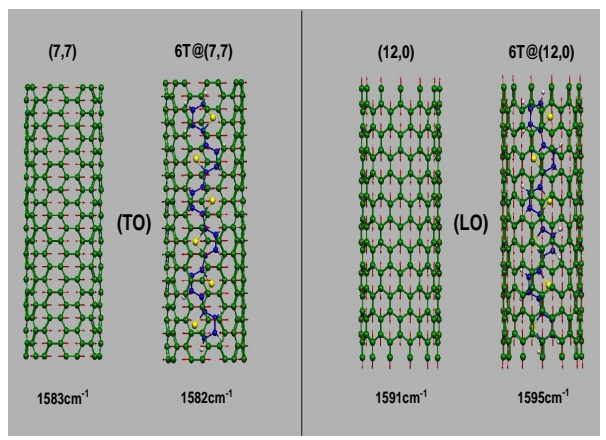

F I G U RE 8 Calculated eigen displacement vectors for the TM in $(7,7)$ and $6 \mathrm{~T} @(7,7)($ left $)$, and $(12,0)$ and 6T@(12,0) (right).

Concerning the high-frequency region of achiral SWNTs where the TM are observed (figures 6 and 7), the calculated ZZ-polarized Raman spectra are featured by a single peak assigned to $A_{1 g}$ (ZZ) TM, centered at $1590 \mathrm{~cm}^{-1}$ in the $(16,0)$ zigzag SWNT, and at $1585 \mathrm{~cm}^{-1}$ in the $(9,9)$ armchair SWNT. The $G^{+}$peak (resp. $G^{-}$) is dominant at the zigzag chiral angle $\left(\theta=0^{\circ}\right)$ (resp. armchair chiral angle $\left(\theta=30^{\circ}\right)$ as discussed in the previous section. For chiral SWNTs, the two considered tubes are $(13,5)$ and $(12,6)$, with $\theta \simeq 15^{\circ}$, and $19^{\circ}$, respectively. Both the $G^{+}(\mathrm{LO})$ and $G^{-}$(TO) peaks appear in the Raman spectra of empty SWNTs.

As we can see in figures 6 and 7, the encapsulation of two molecules does not affect the peaks position of the G-band in the both metallic and semiconducting nanotubes spectra. However, CT between the encapsulated molecules and the
SWNTs causes shifts of the G-band peaks ${ }^{30}$. Thus, since there is no change in the peak positions of the G-band after encapsulation, we conclude that CT does not occur in 6T$6 \mathrm{~T} @$ SWNTs hybrid systems. Indeed, according to Ref ${ }^{31}$, the encapsulation of $\beta$-carotene inside SWNTs (with a diameter of about $1.4 \mathrm{~nm}$ ) leads to an RBM up-shift of $3 \mathrm{~cm}^{-1}$, and none change in the peak position of the G-band could be observed. Besides, Loi et $\mathrm{al}^{37}$ have reported an experimental work on the encapsulation of 6T inside SWNTs whom diameters are between 1.2 and $1.5 \mathrm{~nm}$. The encapsulation of $6 \mathrm{~T}$ gave an RBM up-shift of $5 \mathrm{~cm}^{-1}$ and the G-band frequencies was found to be the same for SWNTs and 6T@SWNTs samples.

\section{4 | DISCUSSION AND CONCLU- SIONS}

Overall, the outstanding optoelectronic properties of semiconducting SWNTs make them highly attractive absorbers integrated in the active layer of OSCs. Their optical band gap can be tuned to optimize absorption of different spectral ranges by tailoring the distribution of their diameters. An efficient absorption of the solar spectrum can be achieved by tuning a distribution of SWNTs ranging from 0.8 to $1.2 \mathrm{~nm}$ in diameter resulting in optical band-gaps ranging from 1.0 to $1.3 \mathrm{eV}$, close to the Shockley-Queisser optimum for a single junction PV device. In this paper, we use an original approach to study the encapsulation of only one 6T molecule and two 6T molecules inside the SWNTs cavity. The dynamical model of the SWNT and 6T molecules was described by molecular mechanics and DFT respectively, whereas the 6T-SWNTs coupling between the subsystems was described by an LJ potential. First, we determined the range of SWNT diameters in which the hybrids are stable for the two encapsulated systems6T@SWNTs and6T-6T@SWNTs. We found that the configurations suitable in terms of the energetics for the insertion of a single 6T molecular chain (resp. two 6T molecules) corresponding to the optimal SWNT diameter around 0.93 $\mathrm{nm}$ (resp. $1.24 \mathrm{~nm}$ ). Then, for the obtained configurations of 6T@SWNTs and 6T-6T@SWNTs hybrid systems with metallic and semiconducting nanotubes, the nonresonant Raman spectra have been calculated using the bond-polarizability model combined with the spectral moment method. The CT 
in the 6T@SWNTs and6T-6T@SWNTs has been investigated by analyzing the Raman-active modes of the $6 \mathrm{~T}$ molecule, as the RBM and G-band modes of SWNTs, before and after encapsulation. The RBM and G-band shift behaviour with a nanotube diameter dependence revealed an agreeable agreement with the reported experimental results. Our calculations testify to the presence of the CT in the 6T@SWNTs encapsulated systems without specifying their direction. It may occur from or to SWNTs, namely, whether SWNTs work as donor or acceptor in6T@SWNTs hybrids. The identification of the CT and its direction in hybrid systems by electronic and optical calculations is the goal of our future works. Alternatively, we predict that CT does not occur in 6T-6T@SWNTs hybrid systems, since there is no change in the peak positions of the G-band after encapsulation. The G-band of 6T-6T@SWNTs nanostructure is unchanged in comparison with the one of pristine SWNTs. One may therefore assume that incorporation of the 6T molecules into SWNTs cavities with diameters above $1.2 \mathrm{~nm}$ does not lead to a modification of nanotube electronic properties. Thus, only the nanotubes with diameters below $1.2 \mathrm{~nm}$ are interesting for OSCs.

Finally, SWNTs with a diameter distribution of 0.9-1nm, appropriate to S1 optical band-gaps in the energy range 1$1.2 \mathrm{eV}$, could be harness for photon capturing with energies above $1 \mathrm{eV}$, which constitute practically the same spectral band trapped by a silicon-based SCs $18 ; 87 ; 15$. Indeed, following light absorption by either SWNTs or 6T molecules (under the concept of BHJs), the excitation of $6 \mathrm{~T}$ accompanied by electron (resp. hole) transfer or the excitation of SWNTs accompanied by hole (resp. electron) transfer, produce the same charge separation state, consequently, the spectral coverage achieved by a certain mixture of SWNTs with average diameters of $0.93 \mathrm{~nm}$ (the optimal value found to encapsulate only one 6T) could be exploited to optimize light-harvesting and, by extension, the PCE of OSCs. In this context, we believe that the encapsulation of photo-active organic molecules inside SWNTs, thus creating a new hybrid system, appear to be of great interest in terms of high PCEs, and long-term stability for commercial application of OSCs.

\section{ACKNOWLEDGMENT}

The work was supported by Moulay Ismail University Research Support (13-16) and Comisión Nacional de Investigación Científica y Tecnológica (CONICYT)-Fondo Nacional de Desarrollo Científico y Tecnológico (FONDECYT) Posdoctodado 2020 No 3200046. JML acknowledges support from Financiamiento basal para centros científicos y tecnológicos de excelencia AFB180001.

\section{CONFLICT OF INTEREST}

The authors declare no conflict of interest.

\section{REFERENCES}

1. K. N. Pabitra, S. Mahesh, H. J. Snaith, D. Cahen, Nature Reviews Materials 2019, 4 (4), 269.

2. W. Shockley, Hans J Queisser, Journal of applied physics 1961, 32 (3), 510-519.

3. M. A. Green, Progress in Photovoltaics: Research and Applications 2009, 17 (3), 183-189.

4. A. Polman, M. Knight, E. C. Garnett, B. Ehrler, W. C. Sinke, Science 2016, 352 (6283), aad4424.

5. G. Li, R. Zhu, Y. Yang, Nature photonics 2012, 6 (3), 153.

6. R. AJ Janssen, J. Nelson, Advanced Materials 2013, 25 (13), 1847-1858.

7. K. Wang, Y. Li, Y. Li, Macromolecular Rapid Communications 2020, 1900437.

8. R. Ganesamoorthy, G. Sathiyan, P. Sakthivel, Solar Energy Materials and Solar Cells 2017, 161, 102-148.

9. W. Tress in Organic Solar Cells, Springer, 2014, pp. 67-214.

10. M. A Green, E. D Dunlop, D. H Levi, J. Hohl-Ebinger, M. Yoshita, A. WY Ho-Baillie, Progress in Photovoltaics: Research and Applications 2019, 27 (7), 565-575.

11. M. C Scharber, Advanced Materials 2016, 28 (10), 1994 2001.

12. H. A. Alturaif, Z. A. ALOthman, J. G. Shapter, S. M. Wabaidur, Molecules 2014, 19 (11), 17329-17344. 
13. S. Cataldo, P. Salice, E. Menna, B. Pignataro, Energy \& Environmental Science 2012, 5 (3), 5919-5940.

14. F. Wang, K. Matsuda in Nanocarbons for Energy Conversion: Supramolecular Approaches, Springer, 2019, pp. 497-536.

15. I. Jeon, Y. Matsuo, S. Maruyama in Single-Walled Carbon Nanotubes, Springer, 2019, pp. 271-298.

16. E. B. Barros, R. B. Capaz, A. Jorio, G. G. Samsonidze, A. G. Souza Filho, S. Ismail-Beigi, C. D. Spataru, S. G. Louie, G. Dresselhaus, M.S. Dresselhaus, Physical Review B 2006, 73 (24), 241406.

17. S. Berciaud, L. Cognet, B. Lounis, Physical Review Letters 2008, 101 (7), 077402.

18. H. Kataura, Y. Kumazawa, Y. Maniwa, I. Umezu, S. Suzuki, Yo. Ohtsuka, Y. Achiba, Synthetic metals 1999, 103 (1-3), 2555-2558.

19. M. Ichida, S. Mizuno, Y. Tani, Y. Saito, A. Nakamura, Journal of the Physical Society of Japan 1999, 68 (10), 31313133 .

20. S. Jin, G. H. Jun, S. Jeon, S. H. Hong, Nano Convergence 2016, $3(1), 8$.

21. M. J. O'connell, S. M. Bachilo, C. B. Huffman, V. C. Moore, M. S. Strano, E. H. Haroz, K. L. Rialon, P. J. Boul, W. H. Noon, C. Kittrell, et al., Science 2002, 297 (5581), 593-596.

22. P. Avouris, M. Freitag, V. Perebeinos, Nature photonics 2008 , $2(6), 341$.

23. T. Dürkop, SA. Getty, E. Cobas, MS. Fuhrer, Nano letters 2004, 4 (1), 35-39.

24. Z. Yao, C. L. Kane, C. Dekker, Physical Review Letters 2000 , 84 (13), 2941.

25. P-H. Hermes, M-P. Gabriela, V-R. Ileana, C. Fusaro, L-V. Fernando, M-A. Mariana, C. Padilla-Rodríguez, F-L. Fabián in Green Nanoparticles, Springer, 2020, pp. 77-115.

26. Y. Kanai, J. C. Grossman, Nano letters 2008, 8 (3), 908-912.

27. J. M. Holt, A. J. Ferguson, N. Kopidakis, B. A. Larsen, J. Bult, G. Rumbles, J. L. Blackburn, Nano letters 2010, 10 (11), 4627-4633.

28. P. Cheng, X. Zhan, Chemical Society Reviews 2016, 45 (9), 2544-2582.

29. M. Karakawa, K. Suzuki, T. Kuwabara, T. Taima, K. Nagai, M. Nakano, T. Yamaguchi, K. Takahashi, Organic Electronics 2020, 76, 105448 .
30. T. Takenobu, T. Takano, M. Shiraishi, Y. Murakami, M. Ata, H. Kataura, Y. Achiba, Y. Iwasa, Nature materials 2003, 2 (10), 683-688.

31. K. Yanagi, Y. Miyata, H. Kataura, Advanced Materials 2006, $18(4), 437-441$.

32. J. Chenouf, M. Boutahir, B. Fakrach, AH. Rahmani, H. Chadli, A. Rahmani, Journal of Raman Spectroscopy 2020, 51 (8), 1315-1323.

33. J. Chenouf, M. Boutahir, B. Fakrach, AH. Rahmani, H. Chadli, P. Hermet, J-M López, A. Rahmani, Journal of Computational Chemistry 2020, 41 (28), 2420-2428.

34. M. Zhang, X. Guo, W. Ma, H. Ade, J. Hou, Advanced Materials 2014, 26 (33), 5880-5885.

35. G. Turkoglu, M. E. Cinar, T. Ozturk in Sulfur Chemistry, Springer, 2019, pp. 79-123.

36. M. Kalbáč, L. Kavan, S. Gorantla, T. Gemming, L. Dunsch, Chemistry-A European Journal 2010, 16 (38), 11753-11759.

37. M. A. Loi, J. Gao, F. Cordella, P. Blondeau, E. Menna, B. Bártová, C. Hébert, S. Lazar, G. A. Botton, M. Milko, Advanced materials 2010, 22 (14), 1635-1639.

38. E. Gaufrès, NY-Wa. Tang, F. Lapointe, J. Cabana, M-A. Nadon, N. Cottenye, F. Raymond, T. Szkopek, R. Martel, Nature Photonics 2014, 8 (1), 72-78.

39. E. Gaufrès, NY-Wa. Tang, A. Favron, C. Allard, F. Lapointe, V. Jourdain, S. Tahir, C-N. Brosseau, R. Leonelli, R. Martel, ACS nano 2016, 10 (11), 10220-10226.

40. C. Fu, M. B. Oviedo, Y. Zhu, A. von Wald Cresce, K. Xu, G. Li, M. E. Itkis, R. C. Haddon, M. Chi, Y. Han, ACS nano 2018, 12 (10), 9775-9784.

41. G. Li, C. Fu, M. B. Oviedo, M. Chen, X. Tian, E. Bekyarova, M. E. Itkis, B. M. Wong, J. Guo, R. C. Haddon, Journal of the American Chemical Society 2016, 138 (1), 40-43.

42. O. V. Sedelnikova, O. A. Gurova, A. A. Makarova, A. D. Fedorenko, A. D. Nikolenko, P. E. Plyusnin, R. Arenal, L. G. Bulusheva, A. V. Okotrub, Nanomaterials 2020, 10 (5), 818.

43. A. Cadena, B. Botka, E. Székely, K. Kamarás, physica status solidi (b) 2020, 2000314.

44. C. Benoit, E. Royer, G. Poussigue, Journal of Physics: Condensed Matter 1992, 4 (12), 3125.

45. J. M. Soler, E. Artacho, J. D. Gale, A. García, J. Junquera, P. Ordejón, D. Sánchez-Portal, Journal of Physics: Condensed Matter 2002, 14 (11), 2745. 
46. G. Dresselhaus, S. Riichiro, Physical properties of carbon nanotubes, World scientific, 1998.

47. S Guha, J Menende, JB Page, GB Adams, Physical Review B 1996, 53 (19), 13106.

48. P Hermet, N Izard, A Rahmani, Ph Ghosez, The Journal of Physical Chemistry B 2006, 110 (49), 24869-24875.

49. A Rahmani, J-L Sauvajol, S Rols, C Benoit, Physical Review B 2002, 66 (12), 125404.

50. A. Rahmani, J-L. Sauvajol, J. Cambedouzou, C Benoit, Physical Review B 2005, 71 (12), 125402.

51. AH. Rahmani, M. Boutahir, A. El Biyaali, B. Fakrach, H. Chadli, K. Sbai, P. Hermet, J-L. Bantignies, A. Rahmani, RSC advances 2016, 6 (47), 41025-41031.

52. M. Boutahir, AH. Rahmani, H. Chadli, A. Rahmani, The European Physical Journal Applied Physics 2016, 74 (2), 24605.

53. L. Alvarez, Y. Almadori, R. Arenal, R. Babaa, T. Michel, R. Le Parc, J-L. Bantignies, B. Jousselme, S. Palacin, P. Hermet, The Journal of Physical Chemistry C 2011, 115 (24), 11898-11905.

54. P. T. Araujo, S. K. Doorn, S. Kilina, S. Tretiak, E. Einarsson, S. Maruyama, H. Chacham, M. A. Pimenta, A. Jorio, Physical Review Letters 2007, 98 (6), 067401.

55. T. Yumura, H. Yamashita, The Journal of Physical Chemistry C 2014, 118 (10), 5510-5522.

56. A. Degli Esposti, M. Fanti, M. Muccini, C. Taliani, G. Ruani, The Journal of Chemical Physics 2000, 112 (13), 5957-5969.

57. G Louarn, JP Buisson, S Lefrant, D Fichou, The Journal of Physical Chemistry 1995, 99 (29), 11399-11404.

58. E. Agosti, M. Rivola, V. Hernandez, M. Del Zoppo, G. Zerbi, Synthetic metals 1999, 100 (1), 101-112.

59. MS Dresselhaus, G Dresselhaus, Ado Jorio, AG Souza Filho, R Saito, Carbon 2002, 40 (12), 2043-2061.

60. M. V. Kharlamova, Progress in Materials Science 2016, 77, 125-211.

61. LA. Girifalco, M. Hodak, R. S. Lee, Physical Review B 2000, $62(19), 13104$.

62. L. A. Girifalco, M. Hodak, Physical Review B 2002, 65 (12), 125404.
63. H. Ulbricht, G. Moos, T. Hertel, Physical review letters 2003, $90(9), 095501$.

64. H. Ulbricht, T. Hertel, The Journal of Physical Chemistry B 2003, 107 (51), 14185-14190.

65. A. N. Khlobystov, R. Scipioni, D. Nguyen-Manh, D. A. Britz, D. G. Pettifor, G. A. D. Briggs, S. G. Lyapin, A. Ardavan, R. J. Nicholas, Applied physics letters 2004, 84 (5), 792-794.

66. B. W. Smith, R. M. Russo, S. B. Chikkannanavar, D. E. Luzzi, Journal of applied physics 2002, 91 (11), 9333-9340.

67. S. Bandow, M. Takizawa, H. Kato, T. Okazaki, H. Shinohara, S. Iijima, Chemical physics letters 2001, 347 (1-3), 23-28.

68. S. Okada, Chemical physics letters 2007, 438 (1-3), 59-62.

69. N. Bendiab, L Spina, A Zahab, P Poncharal, C Marliere, JL Bantignies, E Anglaret, JL Sauvajol, Physical Review B 2001, 63 (15), 153407.

70. N. Bendiab, E. Anglaret, J-L. Bantignies, A. Zahab, JL. Sauvajol, P. Petit, C. Mathis, S. Lefrant, Physical Review B 2001, 64 (24), 245424.

71. G. Chen, CA. Furtado, S. Bandow, S. Iijima, PC. Eklund, Physical Review B 2005, 71 (4), 045408.

72. D. Britz A, A. N. Khlobystov, Chemical Society Reviews 2006, 35 (7), 637-659.

73. H. Farhat, K-I. Sasaki, M. Kalbac, M. Hofmann, R. Saito, M. S. Dresselhaus, J. Kong, Physical review letters 2009, 102 (12), 126804.

74. Y. Almadori, L Alvarez, R. Le Parc, R. Aznar, F. Fossard, A. Loiseau, B. Jousselme, S. Campidelli, P. Hermet, A. Belhboub, et al., The Journal of Physical Chemistry C 2014, 118 (33), 19462-19468.

75. Y. Kinno, H. Omachi, Y. Nakanishi, H. Shinohara, Chemistry Letters 2018, 47 (8), 1022-1025.

76. S-K. Joung, T. Okazaki, N. Kishi, S. Okada, S. Bandow, S. Iijima, Physical review letters 2009, 103 (2), 027403.

77. AM. Rao, E. Richter, S. Bandow, B. Chase, PC. Eklund, KW. Williams, M. Menon, KR. Subbaswamy, A. Thess, RE. Smalley, et al., Science 1997, 275, 187-191.

78. M. S. Dresselhaus, G. Dresselhaus, R. Saito, A. Jorio, Physics reports 2005, 409 (2), 47-99.

79. T. Enoki, M. Suzuki, M. Endo, Graphite intercalation compounds and applications, Oxford University Press, 2003. 
80. P. Corio, AP. Santos, PS. Santos, MLA. Temperini, VW. Brar, MA. Pimenta, MS. Dresselhaus, Chemical Physics Letters 2004, 383 (5-6), 475-480.

81. A. A. Eliseev, M. V. Kharlamova, M. V. Chernysheva, A. V. Lukashin, Y. D. Tretyakov, A. S. Kumskov, N. A. Kiselev, Russian Chemical Reviews 2009, 78 (9), 833.

82. MV Kharlamova, JJ Niu, Applied Physics A 2012, 109 (1), $25-29$.

83. S. Grimm, S. P. Schieß1, Y. Zakharko, M. Rother, M. Brohmann, J. Zaumseil, Carbon 2017, 118, 261-267.

84. M. Kalbac, L. Kavan, L. Dunsch, MS. Dresselhaus, Nano letters 2008, 8 (4), 1257-1264.

85. S. Cambré, B. Schoeters, S. Luyckx, E. Goovaerts, W. Wenseleers, Physical review letters 2010, 104 (20), 207401.

86. H. Kataura, Y. Maniwa, M. Abe, A. Fujiwara, T. Kodama, K. Kikuchi, H. Imahori, Y. Misaki, S. Suzuki, Y. Achiba, Applied Physics A 2002, 74 (3), 349-354.

87. M. S. Arnold, J. L. Blackburn, J. J. Crochet, S. K. Doorn, J. G. Duque, A. Mohite, H. Telg, Physical Chemistry Chemical Physics 2013, 15 (36), 14896-14918. 